\title{
Mild behavioral impairment correlates of cognitive impairments in older adults without dementia: mediation by amyloid
} pathology

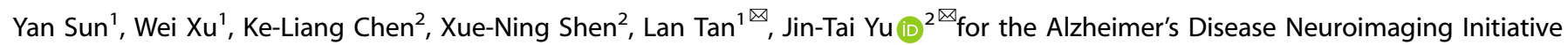

(c) The Author(s) 2021

The relationship between mild behavioral impairment (MBI) and Alzheimer's disease (AD) is intricate and still not well investigated. The purpose of the study is to examine the roles of the $A D$ imaging pathologies in modulating the associations of MBI with cognitive impairments. We analyzed 1129 participants (563 [49.86\%] female), who had measures of Neuropsychiatric Inventory Questionnaire (NPI-Q), cognition, and amyloid PET AD biomarkers from the Alzheimer's disease Neuroimaging Initiative (ADNI). We assess the longitudinal neuropathological and clinical correlates of baseline MBI via linear mixed effects and Cox proportional hazard models. The mediation analyses were used to test the mediation effects of AD pathologies on cognition. We found that MBI was associated with worse global cognition as represented by Mini-Mental State Examination (MMSE) $(p<0.001)$, and higher $\beta$-amyloid burden $(p<0.001)$. $\beta$-amyloid partially mediated the effects of $\mathrm{MBI}$ on cognition with the mediation percentage varied from 14.67 to $40.86 \%$ for general cognition, memory, executive, and language functions for non-dementia individuals. However, no significant associations were discovered between $\mathrm{MBI}$ and tau burden or neurodegeneration. Furthermore, longitudinal analyses revealed that individuals with $\mathrm{MBI}$ had a faster increase in brain amyloid burden $(p<0.001)$ and a higher risk of clinical conversion $(\mathrm{HR}=2.42,95 \% \mathrm{Cl}=1.45$ to $4.01 p<0.001)$. In conclusion, $\mathrm{MBI}$ could be an imperative prediction indicator of clinical and pathological progression. In addition, amyloid pathologies might partially mediate the influences of $\mathrm{MBI}$ on cognitive impairments and $A D$ risk.

Translational Psychiatry (2021)11:577; https://doi.org/10.1038/s41398-021-01675-2

\section{INTRODUCTION}

Characterized by abnormal amyloid deposition, tau phosphorylation and neurodegeneration in pathology and cognitive and behavioral impairments in clinic, Alzheimer's disease (AD) has a prolonged asymptomatic phase in the course of the Alzheimer's continuum before pathological biomarkers appeared [1-4]. Selecting individuals who are at high risk of suffering $A D$ is the critical step to give effect to accurate diagnosis and timely intervention at the early preclinical and prodromal stages of $A D$ and drug development [5]. Neuropsychiatric symptoms (NPSs) mainly manifested as disturbances of mood, perception, and behavior linked with neurodegenerative disease, which are regarded as noncognitive or behavioral and psychiatric symptoms of dementia [6]. NPSs occurred in the prodromal or mild cognitive impairment $(\mathrm{MCl})$ stages of dementia and associated with an increased risk of $A D$ and pathologically associated with greater plaque and tangle burden indicating that preventing NPSs could be a promising way for early intervention [7-9].

The ISTAART-AA criteria for mild behavioral impairment (MBI) has been developed by the International Society to Advance
Alzheimer's Research and Treatment (ISTAART) NPS Professional Interest Area to promote investigation into the correlation between NPSs and dementia [1]. MBI characterized by the new emerged and sustained NPSs may be an early manifestation of neurodegenerative disease. The ISTAART-AA MBI criteria emphasize the importance of a significant change from the person's usual behavior or personality persisting for at least 6 months in the following domains: absence of drive and motivation; emotional dysregulation; impulse dyscontrol; social inappropriateness; and abnormal perception and thought content, assessed individually and collectively for their impact on cognition. In addition, psychiatric illness was explicitly excluded a priori. MBI is easy to capture using several simple rated scales for NPSs [10]. For instance, the Neuropsychiatric Inventory Questionnaire (NPI-Q) could reflect NPI items into MBI domains. MBI therefore provides a flexible and convenient method for selecting a population at higher risk for cognitive decline and dementia.

Previous studies have demontrated that $\mathrm{MBI}$ was a marker of cognitive decline in intact-elder individuals [11, 12] and also associated with AD pathology [13]. However, although MBI and

\footnotetext{
${ }^{1}$ Department of Neurology, Qingdao Municipal Hospital, Qingdao University, Qingdao, China. ${ }^{2}$ Department of Neurology and Institute of Neurology, Huashan Hospital, State Key Laboratory of Medical Neurobiology and MOE Frontiers Center for Brain Science, Shanghai Medical College, Fudan University, Shanghai, China. ${ }^{3}$ The longitudinal data used in preparation for this article were obtained from the Alzheimer's Disease Neuroimaging Initiative (ADNI) database (adni.loni.usc.edu). As such, the investigators within the ADNI contributed to the design and implementation of $A D N I$ and/or provided data but did not participate in the analysis or writing of this report. A complete listing of ADNI

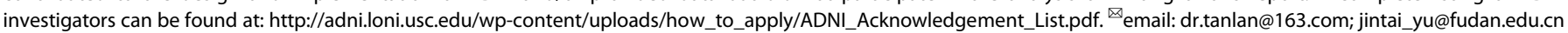


amyloid burden were revealed to contribute to cognitive decline independently, it is still disputable whether amyloid could modulate the relationships of $\mathrm{MBI}$ with cognitive functions. In the current study, we hypothesis that the effects of $\mathrm{MBI}$ on cognition may be modulated by $A D$ pathology in non-dementia elderly individuals. Herein, we test the hypothesis by evaluating the relationships between the $\mathrm{MBI}$ scores, $\mathrm{AD}$ imaging biomarkers including brain burden of $\beta$-amyloid, brain burden of tau and neurodegeneration, and cognitive impairment in participants without dementia. Detecting sensitive and specific markers of very early $A D$ progression is proposed to help develop new treatments and to decrease the time and cost of clinical trials [14].

\section{MATERIALS AND METHODS \\ Subjects}

Data were obtained from the ADNI database (http://adni.loni.usc.edu). The multicenter ADNI project is designed to predict the early onset of AD through investigating clinical, imaging, genetic, and biochemical biomarkers and have been recruited participants from more than 50 sites across the United States and Canada. The participants are older adults aged 55-90 years and their cognitive trajectories and the biomarkers were repeatedly assessed during the follow-up period to track the pathology as the disease progressed. Written informed consent was obtained on human experimentation at each institution and detailed information can be found at http://adni.loni.usc.edu/study-design.

In the study, we selected 1129 participants without dementia, including $543 \mathrm{MCl}$ and $586 \mathrm{CN}$ individuals. The population included ADNI-1 and $A D N I-2$ and $G O$ participants enrolled into the $M B I>0$ and $M B I=0$ cohorts, were tested for positron emission tomography (PET) and NPI-Q.

\section{PET imaging measures}

All PET data used were from the UC Berkeley and Lawrence Berkeley National Laboratory. Florbetapir (AV45) SUVRs calculated by averaging across four cortical regions (frontal, anterior/posterior cingulate, lateral parietal, lateral temporal) were used for brain amyloid burden, and then divided by the whole cerebellum as reference region. Brain tau deposit used a composite metaROI was measured via the flortaucipir (AV-1451) processing method and the bilateral entorhinal, amygdala, fusiform, inferior, and middle temporal regions were considered for tau-PET assessment [15]. Brain neurodegeneration used hypometabolism assessed by $18 \mathrm{~F}$-fluorodeoxyglucose (FDG) PET, which was from the average of five metaROls (left angular gyrus, right angular gyrus, bilateral posterior cingular, left inferior temporal gyrus, right inferior temporal gyrus) [16].

\section{Assessment of mild behavioral impairment}

The $\mathrm{MBI}$ checklist (MBI-C), which was ascertainable instrument for $\mathrm{MBI}$, was not yet incorporated into ADNI [10]. Thus, according to a published algorithm, a transformation of $\mathrm{NPI}-\mathrm{Q}$ scores was approximated to $\mathrm{MBI}$ status [17]. Ten NPSs domains from the NPI-Q were subdivided into five ISTAART-AA MBI domains of the absence of drive and motivation (NPI-Q apathy), emotional dysregulation (NPI-Q depression/anxiety/euphoria), impulse dyscontrol (NPI-Q agression/irritability/aberrant motor behavior), social inappropriateness (NPI-Q disinhibition), and abnormal perception and thought content (NPI-Q delusions/hallucinations). The ISTAART-AA MBI criteria put an emphasis on the necessary of a distinct change from the person's usual behavior or personality persisting for at least 6 months in the five domains $[1,13]$. Finally, adding together the five transformed domains to obtain the approximated MBI status. Participants with an MBI score $>1$ were considered as $\mathrm{MBI}+$ status and participants with an $\mathrm{MBI}$ score of 0 were considered as MBI- status [18]. All individuals were over the age of 55 and none of them met criteria for any major neuropsychiatric disorder.

\section{Cognitive assessment}

We used multiple scales to assess cognitive functions, including the global cognition by Mini-Mental State Examination (MMSE), the Montreal Cognitive Assessment Scale (MoCA) and the cognitive section of Alzheimer's Disease Assessment Scale (ADAS). Participants also underwent neuropsychological evaluation of three cognitive domains (executive, memory and language functions), which were assessed by reviewing the neuropsychological batteries to identify items that could be considered indicators of these three domains $[19,20]$. Above mentioned scales were used to represent general and specific cognition functions.

\section{Statistical analyses}

Data were presented mean (standard deviation, SD) or number (percentage, \%) when appropriate. First, we examined the relationships of $\mathrm{MBI}$ scores with cognition and AD PET imaging biomarkers in different groups. Linear regression models were used to investigate the crosssectional relationships of $\mathrm{MBI}$ scores with PET imaging biomarkers and cognitive measures. Then, the relation between $\mathrm{MBI}$ and clinical progression was tested by calculating cumulative incidence using the Kaplan-Meier method. Clinical progression was defined as: (1) diagnosed as $\mathrm{MCl} /$ dementia for baseline $\mathrm{CN}$ individuals, (2) diagnosed as dementia for baseline $\mathrm{MCl}$ individuals. Cox proportion hazards model was conducted to estimate the hazard ratio (HR) with $95 \%$ confidence interval (Cl). Individuals who did not develop MCl/AD or who were lost were censored at the time of their last evaluation.

Table 1. Baseline characteristics of participants according to groups.

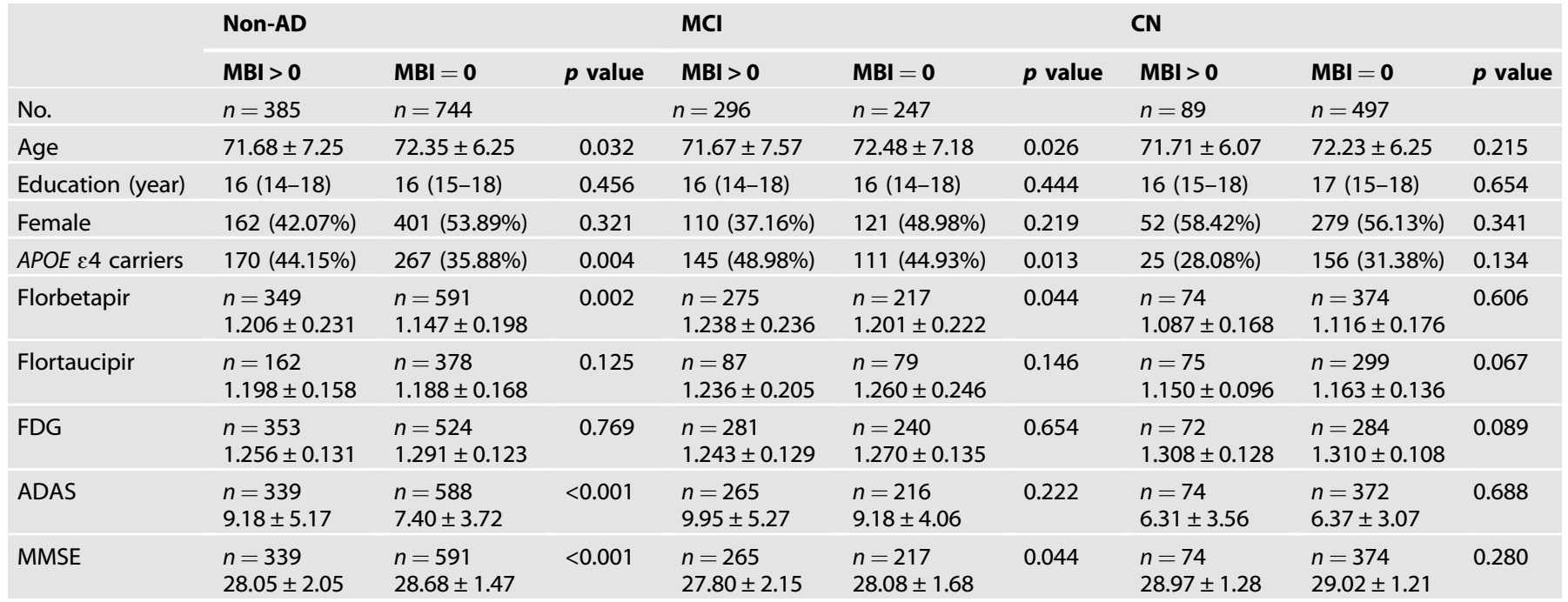

No. number of participants, $M B I$ mild behavioral impairment, $A P O E \varepsilon 4$ apolipoprotein $E$ gene, Non- $A D$ participants without alzheimer's disease, $M C l$ mild cognition impairment, CN cognitively normal, FDG 18F-fluorodeoxyglucose, ADAS Alzheimer's disease assessment scale, MMSE mini-mental state examination, MoCA Montreal cognitive scale. 


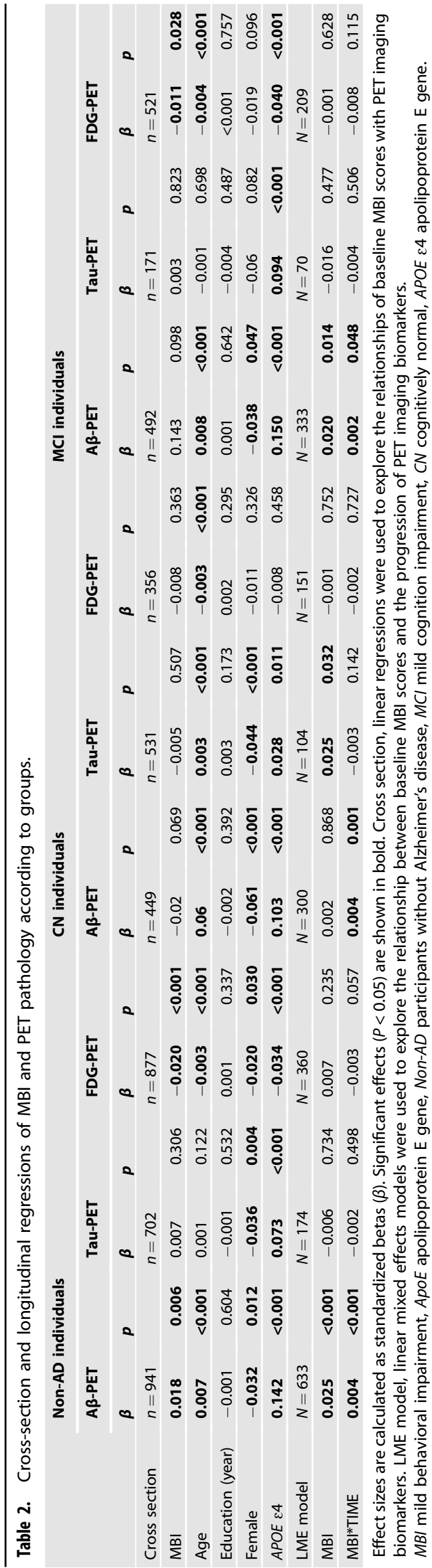

Next, the mediation analyses were used to evaluate whether the association between $\mathrm{MBI}$ and cognition was mediated amyloid pathologies. Baron and Kenny have proposed the calculation methods through linear regression models fitting [21] and we also reference the detail methods in an previous study [22]. The first equation regressed mediator (PET imaging biomarkers) on the independent variable (MBI). The second equation regressed the dependent variable (cognitive scores) on the independent variable. The third equation regressed the dependent variable on both the independent variable and the mediator variable. Diagnosis was not considered as a covariate due to the close relationship between cognitive score and clinical diagnosis ( $\mathrm{MCl}$ vs $\mathrm{CN}$ ) in the analyses with cognitive scores as the dependent variables. The establishment of the mediation effects must satisfy the following criteria simultaneously: (1) MBI was associated with PET imaging biomarkers significantly; (2) MBI was associated with cognitive measures significantly; (3) PET imaging biomarkers were associated with cognitive measures significantly; (4) the association between $\mathrm{MBI}$ and cognition was diminished when PET imaging biomarkers (the mediator) were added in the regression model. In all above analyses, age, gender, diagnosis, education, and APOE4 status were controlled.

Finally, we used the linear mixed effects models to track the longitudinal relationship between $\mathrm{MBI}$ and the imaging biomarkers. The PET imaging biomarkers were considered as the dependent variable and $\mathrm{MBI}$ total scores and $\mathrm{MBI}$ status (MBI scores $>1$ considered as $\mathrm{MBI}+$ while $\mathrm{MBI}$ scores $=0$ considered as $\mathrm{MBI}-$ ) were treated as independent variables. The linear mixed effects models included random intercepts and slopes for time and an unstructured covariance matrix for the random effects, and regarded the interaction between time (continuous) and the dependent variable ( $\mathrm{MBI}+$ vs $\mathrm{MBI}-$ or $\mathrm{MBI}$ total scores) as a predictor. We have adjusted age, gender, education, diagnosis and APOE4 status as covariates in all analyses.

All statistical analyses and figure preparation were conducted on $\mathrm{R}$ software (version 3.5.1). All above analyses were conducted by "Ime4", "survival", "ggplot2", "ggpubr", "magrittr", "survminer", "nlme", "car" and "mediate" packages in R 3.5.1 software. Two-sided $P$ value $<0.05$ were considered as significance.

\section{RESULTS}

\section{Characteristics of participants}

A total of 1129 individuals without dementia $(543 \mathrm{MCl}$ and 586 $\mathrm{CN}$ ) were included into the study. The mean (SD) age of the $\mathrm{MBI}=$ 0 cohorts was 72.35 (6.25) years old and females accounted for $53.89 \%$. MBI $>0$ cohorts have higher proportion of male, APOE4 positive and younger with mean age of 71.68 (7.25), and they had poorer baseline global cognition scores and higher burden of cerebral amyloid burden. Characteristics of participants grouped by baseline diagnosis were summarized in Table 1 .

\section{Associations of MBI with PET imaging biomarkers and cognitive measures}

As Table 2 summarized, in the cross-section, individuals with higher MBI total scores which means more NPSs had greater cerebral amyloid deposition, as indicated by higher $\beta$-amyloid $(\beta=0.018, p=0.006)$ burden and lower FDG PET $(\beta=-0.020$, $p<0.001$ ) in older individuals without dementia. The relationship reminded significant after controlling for age, gender, years of education, diagnosis (in total samples), and APOE4 status. When categorized the total sample into two subgroups, there is no significant findings except the lower FDG PET in $\mathrm{MCl}$ group. Also, no significant associations with tau PET were found in all groups. In addition, non-AD individuals with higher MBI scores had lower global cognitive measures as represented by MMSE $(\beta=-0.113$, $p=0.037)$, MoCA $(\beta=-0.508, p<0.001)$, ADAS $(\beta=0.619$, $p<0.001$ ) (Supplementary Table 1).

\section{Causal mediation analyses}

All the above findings suggested that $\mathrm{MBI}$ was not only an independent risk factor for cognition decline, but also associated with amyloid pathology. To examine whether amyloid pathology was a potential modulator of $\mathrm{MBI}$ on cognition, we further conducted mediation analyses. As the results showed, the 
A

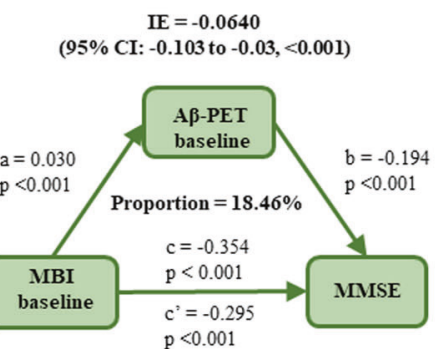

D
B

$$
\text { IE }=-0.1530
$$

(95\% CI: -0.183 to $-0.05,<0.001)$

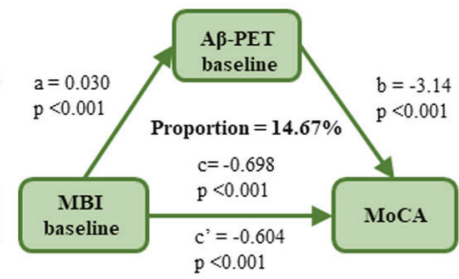

$\mathrm{E}$ (95\% CI: -0.0104 to $0.00,<0.001)$

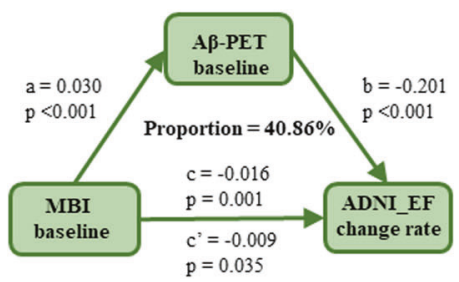

IE $=-0.0070$

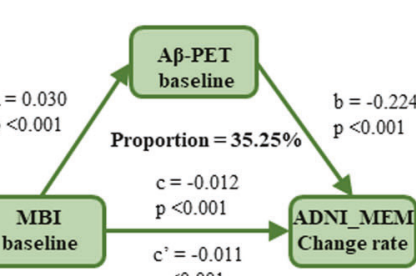

C

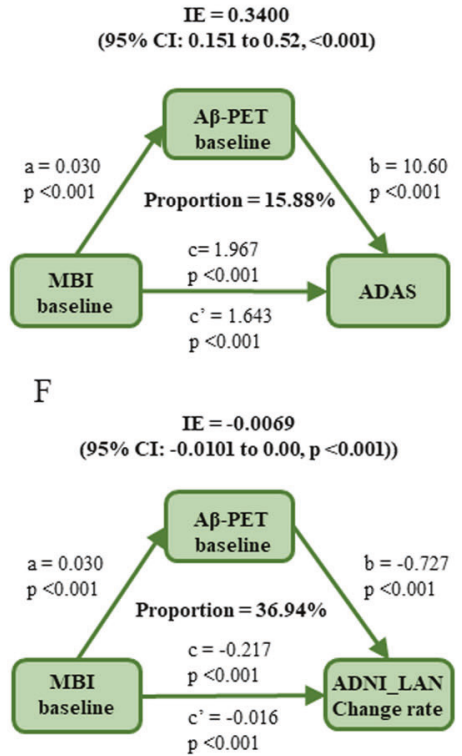

Fig. 1 Mediation analyses with cognitive domains, MMSE, MoCA, and ADAS as cognitive outcomes in non-dementia individuals. The relationship between $\mathrm{MBI}$ and global cognition measured by MMSE (A), MoCA (B), ADAS (C), as well as cognitive domain of executive (D), memory (E) and language (F) function was mediated by $\beta$-amyloid. IE indirect effect. Path a, regression model of $\beta$-amyloid on MBI. Path b, regression model of the cognitive scores on $\beta$-amyloid. Path $\mathrm{c}$, regression model of cognitive scores on MBI. Path $\mathrm{C}^{\prime}$, regression model of cognitive scores on both $\mathrm{MBI}$ and $\beta$-amyloid.

relationship between $\mathrm{MBI}$ and cognitive impairment was mediated mainly by $\beta$-amyloid (Fig. 1). In the total samples, in the first equation, $\mathrm{MBI}$ was significantly associated with higher levels of $\beta$-amyloid deposition $(p=0.033)$. In the second regression, $\mathrm{MBI}$ showed a significant association with poorer global cognition measured by MMSE $(p<0.001)$, MoCA $(p<0.001)$, and ADAS $(p<0.001)$. Finally, in the third equation, when put the amyloid indicator and $\mathrm{MBI}$ simultaneously into the model, we found that the influences of $\mathrm{MBI}$ on global cognition remained but were significantly diminished. The effect was considered partial mediation with the proportion of mediation varying from $14.67 \%$ to $18.46 \%$. The change rates of cognitive domains (memory, executive and language functions) were also calculated. Similar results were concluded for the three functional domains. The proportion of mediation varied from $35.25 \%$ to $40.86 \%(p<0.05)$. These findings further supported the hypothesis that amyloid pathology could at least partially modulate the influences of NPSs on cognitive functions.

When stratified all individuals into $\mathrm{MCl}$ or $\mathrm{CN}$ groups, the mediation effects were not established due to criteria were not simultaneously satisfied (Supplementary Figs. 1 and 2). In MCI group, interestingly, the mediation effects were not qualified, because the second criteria (MBI was associated with cognitive measures significantly) was not reached through the indirect effects calculated in the model were all significant. That probably due to the clinical diagnosis $(\mathrm{MCl}$ or $\mathrm{CN}$ ) were intimate to cognitive scores (MMSE, MoCA etc.), stratifying participants into groups according to diagnosis weaken the relationship between independent variable (MBI) and cognitive scores.

As mentioned above, given the lack of associations between MBI and tau burden and FDG, the mediation relationships of the two $A D$ biomarkers between $\mathrm{MBI}$ and cognitive impairments have not established in the present study (Supplementary Figs. 3 and 4).

\section{Longitudinal relationship between $\mathrm{MBI}$ and amyloid pathology}

From total samples, numbers of individuals who provided the necessary follow-up PET imaging data were detailed in Table 2 and the range and distribution of $\mathrm{MBI}$ scores were also detailed (Supplementary Table 2). In the longitudinal section, controlling for age, sex, education, APOE4 status, clinical diagnosis, participants with higher $\mathrm{MBI}$ total scores predicted higher $\beta$-amyloid deposition in total samples $(n=633, \beta=0.003$, interaction with time $p<0.001$ ). We also found that higher MBI scores displayed faster elevation of $\beta$-amyloid burden in both $\mathrm{MCl}(n=300, \beta=$ 0.004 , interaction with time $p=0.001)$ and $\mathrm{CN}(n=333, \beta=0.002$, interaction with time $p=0.048$ ) groups. Then we categorized the total samples into $\mathrm{MBI}+$ and $\mathrm{MBI}-$ groups. Participants with a baseline $\mathrm{MBI}$ total score of 1 were excluded from the study due to diagnostic uncertainty [18]. As shown in Fig. 2 the burden of cerebral $\beta$-amyloid deposition was significantly higher in $\mathrm{MBI}+$ group compared with individuals of $\mathrm{MBI}$ - status, which indicated the trends of greater annual accumulating rates. Same findings were observed in $\mathrm{CN}$ and $\mathrm{MCl}$ groups. In the present study, no relationships were established in the tau PET and FDG PET.

\section{$M B I$ and incident $A D$ risk}

The survival curves of $\mathrm{MBI}$ status and clinical progression were calculated in participants who provided $\beta$-amyloid data and were exhibited in Fig. 3. Individuals $\mathrm{MBI}+$ group showed higher conversion risk (Supplementary Table 3) in clinical progression, based on $\mathrm{MBI}-$ group. In $\mathrm{CN}$ group, individuals with $\mathrm{MBI}+$ presented a progression rate to MCI/AD dementia of $29.68 \%$ while MBI- individuals presented $12.55 \%(\mathrm{HR}=2.81,95 \% \mathrm{Cl}=1.59-4.96$, $p<0.001)$. As the same, MBI+ individuals $(31.18 \%)$ showed a higher progression rate to $A D$ dementia compared with $\mathrm{MBI}-$ individuals (17.24\%) in $\mathrm{MCl}$ group $(\mathrm{HR}=1.93,95 \% \mathrm{Cl}=1.21$ to 3.06, $p=0.005)$.

\section{DISCUSSION}

The present study provided several lines of evidence for the associations of baseline MBI with Alzheimer's pathologies and clinical progression among non-dementia individuals. Furthermore, it found that the influence of $\mathrm{MBI}$ on cognition was partially mediated by amyloid pathology. As the results showed, individuals 

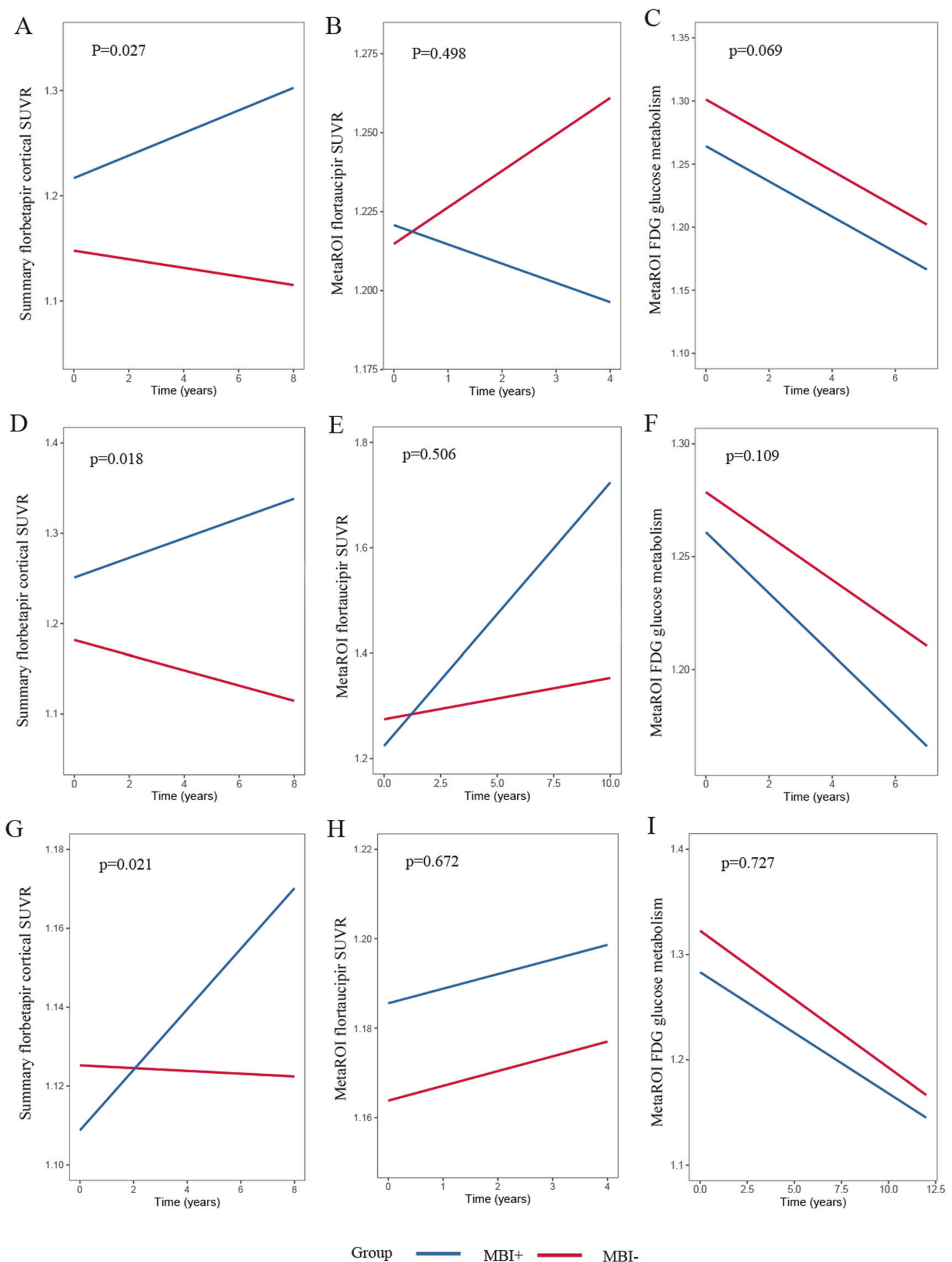

Fig. 2 Longitudinal changes of amyloid, tau, and neurodegeneration according to MBI status. ABC Longitudinal changes of $\beta$-amyloid, tau, and neurodegeneration in non-AD individuals; DEF Longitudinal changes of $\beta$-amyloid, tau, and neurodegeneration in $\mathrm{MCl}$ individuals; GHI Longitudinal changes of $\beta$-amyloid, tau, and neurodegeneration in $\mathrm{CN}$ individuals.

with $\mathrm{MBI}$ possessed a faster accumulation of brain amyloidosis and a higher risk of clinical conversion, suggesting a predictive link of $\mathrm{MBI}$ with amyloidosis progression. In addition, higher MBI scores, as reflected by serious NPSs, predicted a more severe future progress. All these findings support that $\mathrm{MBI}$ can provide imperative information for early detection and intervention. Base on the above findings, it could be inferred that cerebral amyloid deposition could mediate the effects of $\mathrm{MBI}$ on cognitive impairment.
The present study further verified that the predictive relationship of baseline $\mathrm{MBI}$ in non-dementia individuals with the progress of amyloid pathology and firstly revealed the relationship of $\mathrm{MBI}$ and cognitive impairment may via amyloid pathology. It crucially replenishes the gap in current research and further elaborates on the impact of NPSs on subsequent pathological and clinical progression. The previous study also suggested that drugs either reducing amyloid, preventing tau hyperphosphorylation, or 

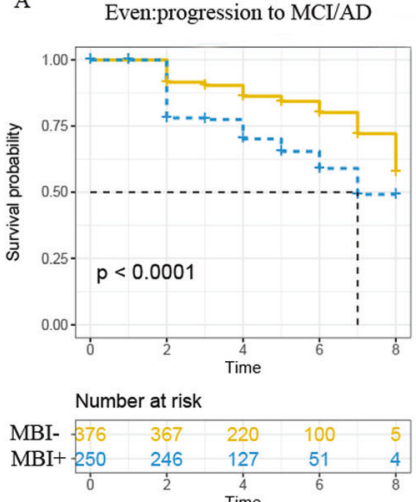

B

Event:progress from MCI to $\mathrm{AD}$
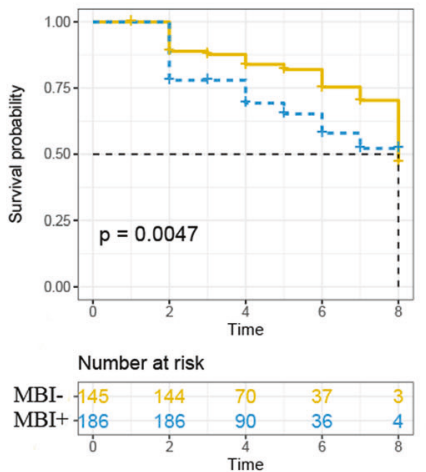
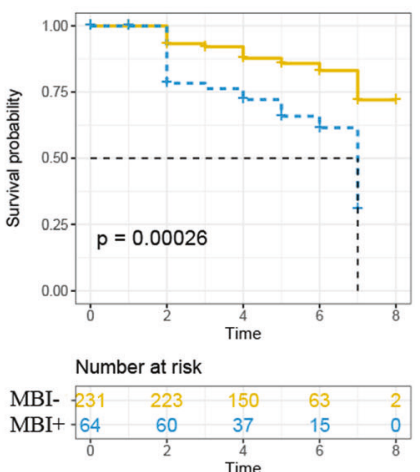

Fig. 3 Kaplan-Meier curves showing survival probability of clinical progression according to groups. A Progression from $C N$ to $M C I$ and progression from $\mathrm{MCl}$ to $\mathrm{AD}$ dementia. B Progression from $\mathrm{MCl}$ to $\mathrm{AD}$ dementia. C Progression from $\mathrm{CN}$ to $\mathrm{MCl}$ or $\mathrm{AD}$ dementia.

modifying neurodegeneration were reliable for earlier intervention through feasible methods for early detection of preclinical AD [13].

$M B I$ has been identified to be indicators of preclinical $A D$ previously $[2,12,23]$. A recent review about the role of NPSs in diagnostic criteria for $A D$ dementia has explained that $M B I$ is the harbinger of a progressive dementia syndrome occurred with $\mathrm{MCl}$ or not [24]. It was reported as a representation of the neurobehavioral axis of pre-dementia risk states and complemented the neurocognitive axis represented by subjective cognitive decline and $\mathrm{MCl}$. Actually, clinical and imaging biomarker studies have generally accepted that $\mathrm{MBI}$ is a risk state and marker of early disease [18]. Previous research has validated MBI as a preclinical dementia syndrome and associated with amyloid positivity, before tau and neurodegeneration in older adults with normal cognition, which was consistent with our findings that $\mathrm{MBI}$ was a cause to the cognitive decline mainly via $\beta$-amyloid, also in advance of tau or neurodegeneration biomarkers, irrespective of the presence or absence of subjective cognitive complaints [13]. In addition, the relationship between dementia amyloid pathologies and NPSs have also been supported by recent studies. For instance, NPSs such as anxiety can be further investigated by staging $\beta$-amyloid deposition in both cortical and subcortical regions [25], and previous study has also reported that increased depressionanxiety scores correlated to elevated cortical amyloid deposition [26]. All these findings supposed the synergetic roles of MBI and amyloid pathology, which was consistent with the mentioned hypothesis. In the present study, we not only revealed that MBI effected cognitive function regardless of amyloid burden, but also found a mediation effect of amyloid pathology.

We did not find the influences of $\mathrm{MBI}$ on cognitive functions were mediated by tau or neurodegeneration. The hypothesis of the present study is that MBI was associated with early but not with later-stage AD pathophysiology [27]. Though considering that tau pathologies could lead to cognitive decline independent of amyloid, significant tau aggregation is rarely observed in cognitive intact individuals [13]. With regard to the neurodegeneration biomarker category, it is reported that early neurodegeneration related loss of biogenic amine nuclei can potentially manifest as psychiatric symptomatology [28]. The negative findings here might be explained as considering the lack of association between $\mathrm{MBI}$ and tau burden and the temporal ordering of $A D$-related pathologies. It has been reported that $A \beta$ pathophysiological processes became abnormal first and then downstream neuronal injury biomarkers, such as tau pathology and neurodegeneration markers became abnormal later [29-31]. But interestingly, in the cross-sectional analyses, we find that higher $\mathrm{MBI}$ scores corresponding to lower FDG-PET, which has been detected in other studies that pathways promoting $A \beta$ and neurodegeneration may initially occur independently [32-34]. All above implied a hierarchical ranking of $A \beta$ biomarkers over downstream neuronal injury biomarkers, hence, our result is not unexpected for the non-dementia individuals.

The etiology of $\mathrm{MBI}$ is still unclear and the mechanisms by which $\mathrm{MBI}$ was involved in regulating amyloid pathology are not wholly explained either. Prior study reported that depression, one of the mainly symptom of the "emotional dysregulation" domain [35], could increase neuroinflammatory cytokines and disorder brain-derived neurotrophic factor by effecting hypothalamicpituitary-adrenal axis altered gamma-amino butyric acid system and then provided a possible pathway hypothesis [36]. Another study showed that NPSs were strongly linked to 3-methoxy-4hydroxyphenylethyleneglycol, which was the intraneuronal metabolite of norepinephrine, and p-tau, that suggesting that the locus coeruleus -norepinephrine may be pivotal to understand connections between $A D$ pathology and behavioral deficits in AD [37]. Actually, though amyloid pathology and $\mathrm{MBI}$ as potential target of early intervention to prevent $A D$ respectively, their interactions still warrant further investigation.

There are limitations in this study. First, the specificity of estimating $\mathrm{MBI}$ via NPI-Q was lower compared to those with the $\mathrm{MBI}-\mathrm{C}$, because the short reference range can result in poor specificity, inappropriately capturing as cases subjects with transient symptoms and reactive conditions, and therefore, resulting in an inflated prevalence estimate $[17,38]$. To elevate the accuracy of this approach to capture $\mathrm{MBI}$, when stratified the $\mathrm{MBI}$ status as categorical variable, score of 1 were excluded, it may prevent to categorize some participants as $\mathrm{MBI}+$ falsely. In addition, MBI affected multi-types of dementia [1, 39] and specificity need to be of concerns. Further researches are required to detect the specificity of $\mathrm{MBI}$ on multi-types of dementia.

\section{CONCLUSIONS}

In conclusion, the present study indicated that amyloid pathology was not only contributed from $\mathrm{MBI}$, but also a key mediator for influences of $\mathrm{MBI}$ on cognitive impairments and $A D$ risk. Examining neurobehavioral outcomes (e.g., mood, social interaction) was highly recommended to link the pathological process to clinical symptoms, which contribute to delineate a time course and depict the impact of it on functional decline prior to overt symptom onset $[40,41]$. These findings supported the hypothesis that $\mathrm{MBI}$ represent an early manifestation of preclinical $\mathrm{AD}$ and could be used to help define high-risk population who are suitable for early prevention of the disease. 


\section{DATA AVAILABILITY}

The dataset generated and analyzed in the current study is available from the corresponding author on reasonable request.

\section{REFERENCES}

1. Ismail Z, Smith EE, Geda Y, Sultzer D, Brodaty H, Smith G, et al. Neuropsychiatric symptoms as early manifestations of emergent dementia: provisional diagnostic criteria for mild behavioral impairment. Alzheimer's Dement. 2016;12:195-202.

2. Burhanullah MH, Tschanz JT, Peters ME, Leoutsakos JM, Matyi J, Lyketsos CG, et al. Neuropsychiatric symptoms as risk factors for cognitive decline in clinically normal older adults: the Cache County Study. Am J Geriatr Psychiatry. 2020;28:64-71.

3. Geda YE, Schneider LS, Gitlin LN, Miller DS, Smith GS, Bell J, et al. Neuropsychiatric symptoms in Alzheimer's disease: past progress and anticipation of the future. Alzheimer's Dement. 2013;9:602-8.

4. Scheltens P, Blennow K, Breteler MM, de Strooper B, Frisoni GB, Salloway S, et al. Alzheimer's disease. Lancet. 2016;388:505-17.

5. Hansson O, Seibyl J, Stomrud E, Zetterberg H, Trojanowski JQ, Bittner T, et al. CSF biomarkers of Alzheimer's disease concord with amyloid- $\beta$ PET and predict clinical progression: a study of fully automated immunoassays in BioFINDER and ADNI cohorts. Alzheimer's Dement. 2018;14:1470-81.

6. Lyketsos CG, Carrillo MC, Ryan JM, Khachaturian AS, Trzepacz P, Amatniek J, et al. Neuropsychiatric symptoms in Alzheimer's disease. Alzheimer's Dement. 2011;7:532-9.

7. Diniz BS, Butters MA, Albert SM, Dew MA, Reynolds CF 3rd. Late-life depression and risk of vascular dementia and Alzheimer's disease: systematic review and meta-analysis of community-based cohort studies. $\mathrm{Br} J$ Psychiatry. 2013;202:329-35.

8. Zubenko GS, Moossy J, Martinez AJ, Rao G, Claassen D, Rosen J, et al. Neuropathologic and neurochemical correlates of psychosis in primary dementia. Arch Neurol. 1991:48:619-24.

9. Bensamoun D, Guignard R, Furst AJ, Derreumaux A, Manera V, Darcourt J, et al. Associations between neuropsychiatric symptoms and cerebral amyloid deposition in cognitively impaired elderly people. J Alzheimer's Dis. 2016;49:387-98.

10. Ismail $\mathrm{Z}$, Agüera-Ortiz L, Brodaty $\mathrm{H}$, Cieslak A, Cummings J, Fischer $C E$, et al. The Mild Behavioral Impairment Checklist (MBI-C): a rating scale for neuropsychiatric symptoms in pre-dementia populations. J Alzheimer's Dis. 2017;56:929-38.

11. Creese B, Brooker H, Ismail Z, Wesnes KA, Hampshire A, Khan Z, et al. Mild behavioral impairment as a marker of cognitive decline in cognitively normal older adults. Am J Geriatr Psychiatry. 2019;27:823-34.

12. Matsuoka T, Ismail Z, Narumoto J. Prevalence of mild behavioral impairment and risk of dementia in a psychiatric outpatient clinic. J Alzheimer's Dis. 2019;70:505-13.

13. Lussier FZ, Pascoal TA, Chamoun M, Therriault J, Tissot C, Savard M, et al. Mild behavioral impairment is associated with $\beta$-amyloid but not tau or neurodegeneration in cognitively intact elderly individuals. Alzheimer's Dement. 2020;16:192-9.

14. Mattsson N, Cullen NC, Andreasson U, Zetterberg H, Blennow K. Association between longitudinal plasma neurofilament light and neurodegeneration in patients with Alzheimer Disease. JAMA Neurol. 2019;76:791-9.

15. Meyer PF, Pichet Binette A, Gonneaud J, Breitner JCS, Villeneuve S. Characterization of Alzheimer disease biomarker discrepancies using cerebrospinal fluid phosphorylated tau and AV1451 positron emission tomography. JAMA Neurol. 2020;77:508-16

16. Landau SM, Harvey D, Madison CM, Koeppe RA, Reiman EM, Foster NL, et al Associations between cognitive, functional, and FDG-PET measures of decline in AD and MCl. Neurobiol Aging. 2011;32:1207-18.

17. Sheikh F, Ismail Z, Mortby ME, Barber P, Cieslak A, Fischer K, et al. Prevalence of mild behavioral impairment in mild cognitive impairment and subjective cognitive decline, and its association with caregiver burden. Int Psychogeriatr. 2018;30:233-44

18. Naude JP, Gill S, Hu S, McGirr A, Forkert ND, Monchi O, et al. Plasma neurofilament light: a marker of neurodegeneration in mild behavioral impairment. J Alzheimer's Dis. 2020;76:1017-27.

19. Gibbons LE, Carle AC, Mackin RS, Harvey D, Mukherjee S, Insel P, et al. A composite score for executive functioning, validated in Alzheimer's Disease Neuroimaging Initiative (ADNI) participants with baseline mild cognitive impairment. Brain Imaging Behav. 2012;6:517-27.

20. Crane PK, Carle A, Gibbons LE, Insel P, Mackin RS, Gross A, et al. Development and assessment of a composite score for memory in the Alzheimer's Disease Neuroimaging Initiative (ADNI). Brain Imaging Behav. 2012;6:502-16.

21. Baron RM, Kenny DA. The moderator-mediator variable distinction in social psychological research: conceptual, strategic, and statistical considerations. J Personal Soc Psychol. 1986;51:1173-82.
22. Xu W, et al. Amyloid pathologies modulate the associations of minimal depressive symptoms with cognitive impairments in older adults without dementia. Biol Psychiatry. 2020;89:766-75.

23. Rouse HJ, Small BJ, Schinka JA, Loewenstein DA, Duara R, Potter H. Mild behavioral impairment as a predictor of cognitive functioning in older adults. Int Psychogeriatr. 2020;33:1-9.

24. Cummings J. The role of neuropsychiatric symptoms in research diagnostic criteria for neurodegenerative diseases. Am J Geriatr Psychiatry. 2020;29:375-83.

25. Hanseeuw BJ, Jonas V, Jackson J, Betensky RA, Rentz DM, Johnson KA, et al. Association of anxiety with subcortical amyloidosis in cognitively normal older adults. Mol Psychiatry. 2018;25:2644.

26. Krell-Roesch J, Lowe VJ, Neureiter J, Pink A, Roberts RO, Mielke MM, et al. Depressive and anxiety symptoms and cortical amyloid deposition among cognitively normal elderly persons: the Mayo Clinic Study of Aging. Int Psychogeriatr. 2018;30:245-51.

27. Tsunoda K, Yamashita T, Osakada Y, Sasaki R, Tadokoro K, Matsumoto N, et al. Early emergence of neuropsychiatric symptoms in cognitively normal subjects and mild cognitive impairment. J Alzheimer's Dis. 2020;73:209-15.

28. Šimić G, Babić Leko M, Wray S, Harrington CR, Delalle I, Jovanov-Milošević N, et al. Monoaminergic neuropathology in Alzheimer's disease. Prog Neurobiol. 2017;151:101-38.

29. McKhann GM, Knopman DS, Chertkow H, Hyman BT, Jack CR Jr, Kawas CH, et al. The diagnosis of dementia due to Alzheimer's disease: recommendations from the National Institute on Aging-Alzheimer's Association workgroups on diagnostic guidelines for Alzheimer's disease. Alzheimer's Dement. 2011;7:263-9.

30. Jack CR Jr, Knopman DS, Jagust WJ, Shaw LM, Aisen PS, Weiner MW, et al. Hypothetical model of dynamic biomarkers of the Alzheimer's pathological cascade. Lancet Neurol. 2010;9:119-28.

31. Wirth $M$, Oh $H$, Mormino EC, Markley C, Landau SM, Jagust WJ. The effect of amyloid $\beta$ on cognitive decline is modulated by neural integrity in cognitively normal elderly. Alzheimer's Dement. 2013;9:687-98. e1

32. Mormino EC, Betensky RA, Hedden T, Schultz AP, Amariglio RE, Rentz DM, et al. Synergistic effect of $\beta$-amyloid and neurodegeneration on cognitive decline in clinically normal individuals. JAMA Neurol. 2014;71:1379-85.

33. Jack CR Jr, Wiste HJ, Weigand SD, Knopman DS, Lowe V, Vemuri P, et al. Amyloidfirst and neurodegeneration-first profiles characterize incident amyloid PET positivity. Neurology. 2013;81:1732-40.

34. Knopman DS, Jack CR Jr, Wiste HJ, Weigand SD, Vemuri P, Lowe VJ, et al. Brain injury biomarkers are not dependent on $\beta$-amyloid in normal elderly. Ann Neurol. 2013;73:472-80.

35. Yoon EJ, Ismail Z, Hanganu A, Kibreab M, Hammer T, Cheetham J, et al. Mild behavioral impairment is linked to worse cognition and brain atrophy in Parkinson disease. Neurology. 2019;93:e766-e77.

36. Caraci F, Spampinato SF, Morgese MG, Tascedda F, Salluzzo MG, Giambirtone MC et al. Neurobiological links between depression and AD: The role of TGF$\beta 1$ signaling as a new pharmacological target. Pharmacol Res. 2018;130:374-84.

37. Jacobs HIL, Riphagen JM, Ramakers I, Verhey FRJ. Alzheimer's disease pathology: pathways between central norepinephrine activity, memory, and neuropsychiatric symptoms. Mol Psychiatry. 2019;26:897-906.

38. Mortby ME, Ismail Z, Anstey KJ. Prevalence estimates of mild behavioral impairment in a population-based sample of pre-dementia states and cognitively healthy older adults. Int Psychogeriatr. 2018;30:221-32.

39. Liu W, Miller BL, Kramer JH, Rankin K, Wyss-Coray C, Gearhart R, et al. Behavioral disorders in the frontal and temporal variants of frontotemporal dementia. Neurology. 2004;62:742-8.

40. Babulal GM, Ghoshal N, Head D, Vernon EK, Holtzman DM, Benzinger TLS, et al Mood changes in cognitively normal older adults are linked to alzheimer disease biomarker levels. Am J Geriatr Psychiatry. 2016;24:1095-104.

41. Sperling RA, Aisen PS, Beckett LA, Bennett DA, Craft S, Fagan AM, et al. Toward defining the preclinical stages of Alzheimer's disease: recommendations from the National Institute on Aging-Alzheimer's Association workgroups on diagnostic guidelines for Alzheimer's disease. Alzheimer's Dement. 2011;7:280-92.

\section{ACKNOWLEDGEMENTS}

We would like to thank all the researchers and participants in the ADNI initiative. Data collection and sharing for ADNI data section was funded by the Alzheimer's Disease Neuroimaging Initiative (ADNI) (National Institutes of Health Grant U01 AG024904) and DOD ADNI (Department of Defense award number W81XWH-12-2-0012). ADNI is funded by the National Institute on Aging, the National Institute of Biomedical Imaging and Bioengineering, and through generous contributions from the following: AbbVie, Alzheimer's Association; Alzheimer's Drug Discovery Foundation; Araclon Biotech; BioClinica, Inc.; Biogen; Bristol-Myers Squibb Company; CereSpir, Inc.; Cogstate; Eisai Inc.; Elan Pharmaceuticals, Inc.; Eli Lilly and Company; Eurolmmun; F. Hoffmann-La Roche Ltd and its affiliated company Genentech, Inc.; Fujirebio; GE 
Healthcare; IXICO Ltd.; Janssen Alzheimer Immunotherapy Research \& Development, LLC.; Johnson \& Johnson Pharmaceutical Research \& Development LLC.; Lumosity; Lundbeck; Merck \& Co., Inc.; Meso Scale Diagnostics, LLC.; NeuroRx Research; Neurotrack Technologies; Novartis Pharmaceuticals Corporation; Pfizer Inc.; Piramal Imaging; Servier; Takeda Pharmaceutical Company; and Transition Therapeutics. The Canadian Institutes of Health Research is providing funds to support ADNI clinical sites in Canada. Private sector contributions are facilitated by the Foundation for the National Institutes of Health (www.fnih.org). The grantee organization is the Northern California Institute for Research and Education, and the study is coordinated by the Alzheimer's Therapeutic Research Institute at the University of Southern California. ADNI data are disseminated by the Laboratory for Neuro Imaging at the University of Southern California. This study was supported by grants from the National Natural Science Foundation of China (91849126), the National Key R\&D Program of China (2018YFC1314700), Shanghai Municipal Science and Technology Major Project (No. 2018SHZDZX01), ZJLab, Shanghai Center for Brain Science and Brain-Inspired Technology, Tianqiao and Chrissy Chen Institute, and the State Key Laboratory of Neurobiology and Frontiers Center for Brain Science of Ministry of Education, Fudan University.

\section{AUTHOR CONTRIBUTIONS}

JTY conceptualized the study, analyzed and interpreted the data, and drafted and revised the manuscript. YS, WX, KLC, and XNS analyzed and interpreted the data, drafted and revised the manuscript, did the statistical analysis, and prepared all the figures. LT interpretation of the data and revision of the manuscript. All authors read and approved the final manuscript.

\section{COMPETING INTERESTS}

The authors declare no competing interests.

\section{ADDITIONAL INFORMATION}

Supplementary information The online version contains supplementary material available at https://doi.org/10.1038/s41398-021-01675-2.

Correspondence and requests for materials should be addressed to Lan Tan or Jin-Tai Yu.

Reprints and permission information is available at http://www.nature.com/ reprints

Publisher's note Springer Nature remains neutral with regard to jurisdictional claims in published maps and institutional affiliations.

(i) Open Access This article is licensed under a Creative Commons Attribution 4.0 International License, which permits use, sharing, adaptation, distribution and reproduction in any medium or format, as long as you give appropriate credit to the original author(s) and the source, provide a link to the Creative Commons license, and indicate if changes were made. The images or other third party material in this article are included in the article's Creative Commons license, unless indicated otherwise in a credit line to the material. If material is not included in the article's Creative Commons license and your intended use is not permitted by statutory regulation or exceeds the permitted use, you will need to obtain permission directly from the copyright holder. To view a copy of this license, visit http://creativecommons. org/licenses/by/4.0/.

(c) The Author(s) 2021 